\title{
Effet de la densité de mise en charge sur les performances zootechniques et la production chez les alevins de Parachanna obscura élevés en milieu contrôlé
}

\author{
Diane N.S. Kpogue Gangbazo1,5, Hervé B. Dakpogan², Arsène F. M. D'almeida ${ }^{3}$, Juste V. D. S. \\ Vodounnou ${ }^{5}$, Germain Aissetche ${ }^{5}$, Guy A. Mensah ${ }^{4}$ Et Emile D. Fiogbe ${ }^{5}$ \\ ${ }^{1} E$ Ecole d'Aquaculture, Université Nationale d'Agriculture, BP 43 Kétou, Bénin \\ 'Ecole de Gestion et d'Exploitation des Systèmes d'Élevage BP 43 Kétou, Bénin \\ ${ }^{3}$ Direction de la Production Halieutique, Ministère de l'Agriculture, de l'Élevage et de la Pêche, 03 BP 618 Cotonou, Bénin \\ ${ }^{4}$ Institut National des Recherches Agricoles du Bénin, Centre de Recherches Agricoles d'Agonkanmey.01 BP 884. \\ Recette Principale Cotonou 01, Bénin. \\ 5 Unité de Recherches sur les Zones Humides, Département de Zoologie, Faculté des Sciences et Techniques, Université \\ d'Abomey - Calavi, B.P. 526 Cotonou, Bénin. \\ *Corresponding author, E-mail : senami_diane@yahoo.fr, Tél. : (+229) 96071407
}

Original submitted in on $18^{\text {th }}$ April 2018. Published online at www.m.elewa.org on $31^{\text {st }}$ August 2018 https://dx.doi.org/10.4314/jab.v128i1.2

\section{RÉSUMÉ}

Objectifs : Cette étude vise à évaluer les effets de la densité de mise en charge des alevins de Parachanna obscura élevés en captivité sur leurs performances zootechniques et leur production.

Méthodologie et résultats : Les densités de 10,15, 20 et 25 alevins / $\mathrm{dm}^{3}$ d'eau ont été testées sur les alevins de $P$. obscura de poids moyen initial $9,6 \mathrm{~g}$ nourris au Coppens pendant huit semaines. Chaque densité a été testée en triplicat. Les résultats obtenus montrent que la densité de mise en charge n'a pas eu d'effet significatif sur le taux de survie qui a été de $100 \%$ pour tous les traitements appliqués. Cependant, les paramètres de croissance et d'utilisation des aliments ont significativement varié en fonction des traitements ( $P$ $<0,05)$. Le taux de croissance spécifique, l'efficacité alimentaire et la production les plus élevés ont été obtenus avec la densité de 25 alevins / $\mathrm{dm}^{3}$ d'eau.

Conclusion et application : Les alevins de $P$. obscura sont très résistants et peuvent être stockés à une densité optimale de 25 alevins / $\mathrm{dm}^{3}$ d'eau afin d'augmenter la production et la rentabilité technique et économique des entreprises aquacoles.

Mots clés : Parachanna obscura, alevins, densité, performances zootechniques, production

Effect of stocking density on zootechnical performances and production of Parachanna obscura fingerlings reared in captivity

\section{ABSTRACT}

Objective : The aim of this study is to evaluate the effects of stocking density on zootechnical performances and production of $P$. obscura fingerlings reared in captivity. 
Methodology and results : Four stocking densities $\left(10,15,20\right.$ and 25 fingerlings / $\left.\mathrm{dm}^{3}\right)$ were tested on $P$. obscura fingerlings during 8 weeks. Fish with initial body weight $9.6 \mathrm{~g}$ were feeding with Coppens. Each treatment was assigned in triplicate. Results showed that in all treatments, survival rate were $100 \%$ and were not significantly affected by stocking density. However, growth and feed utilization parameters were significantly influenced by stocking density $(P<0.05)$. Highest specific growth rate, feed efficiency and production were obtained with stocking density 25 fingerlings/ $\mathrm{dm}^{3}$.

Conclusion and application : $P$. obscura fingerlings are very tough. So, in order to improve production and technical/economical rentability in aquaculture, the optimum stocking density of $P$. obscura fingerlings is 25 alevins $/ \mathrm{dm}^{3}$ in reared conditions.

Keywords : Parachanna obscura, stocking density, zootechnical performances, production.

\section{INTRODUCTION}

La consommation des produits piscicoles tend à augmenter de nos jours pour des questions de goût, des raisons diététiques ou de santé. En effet, la chair de poisson contient peu de cholestérol mais beaucoup d'acides gras mono ou polyinsaturés dont le rôle préventif et protecteur contre les maladies cardio-vasculaires a été démontré par plusieurs études (Regost et al., 2001 ; Cahu et al., 2004). Face à cette situation, le développement de la pisciculture accompagnée d'une diversification des espèces piscicoles élevées paraît évidemment être la meilleure solution. Une diversification reposant sur la domestication d'espèces piscicoles autochtones aux zones d'élevage concernées doit permettre de limiter certains risques environnementaux tout en s'adaptant mieux aux exigences des marchés locaux. Certaines espèces à grandes potentialités aquacoles dont Parachanna obscura ont été alors identifiées (Kpoguè et al., 2013a). En effet, $P$. obscura, le plus répandu des Channidae africains (Bonou et Teugels, 1985), a une grande valeur commerciale et une bonne qualité gastronomique (O'Bryen et Lee, 2007). Sa production par les plans d'eaux naturels continentaux n'arrive plus à couvrir les demandes et son élevage en système extensif a déjà commencé dans certains pays africains (FAO, 2007). Son élevage en système intensif permettra de

\section{MATÉRIEL ET MÉTHODES}

Origine des poissons et conditions expérimentales : Les alevins de $P$. obscura utilisés au cours de cette expérimentation ont été capturés à l'aide d'une nasse dans un marécage nommé «Dra» situé à Takon dans la commune de Sakété (Sud - Est du Bénin). Après leur capture, les alevins ont été transportés à la Station de conserver, renforcer les stocks naturels et produire continuellement des spécimens de taille commerciale destinés à la vente sur les marchés. Dans les systèmes d'élevage intensifs, plusieurs facteurs à savoir la quantité et la qualité de l'aliment (Erondu et al., 2006) et la densité de mise en charge des poissons (Schram et al., 2006 ; Wirat, 2011; Chattopadhyay et al., 2012; Tan et al., 2018) influencent le taux de survie, la croissance, l'efficacité alimentaire et la production. La connaissance des besoins nutritionnels et des densités optimaux de mise en charge des alevins permettent d'améliorer la production et la rentabilité économique en aquaculture (Fiogbé et Kestemont, 2003; Aksungur et al., 2007; de Oliveira et al., 2012 ). Le stockage à des densités trop faibles ou très élevées constitue un facteur de stress chronique qui réduit les performances zootechniques de certaines espèces de poissons (Leatherland et Cho, 1985 ; Ma et al., 2006, Chambel et al., 2015). Les besoins nutritionnels des alevins de $P$. obscura ont été déjà estimés par Kpoguè et Fiogbé (2012) et Kpoguè et al. (2013b, 2013c, 2018). Aucun travail n'ayant encore été réalisé sur l'effet de la densité de mise en charge des alevins de $P$. obscura sur leurs performances zootechniques, cette étude a été alors initiée.

Recherche en Aquaculture de l'Unité de Recherche sur les Zones Humides de la Faculté des Sciences et Techniques de l'Université d'Abomey - Calavi où ils ont été prégrossis. L'expérience a été conduite pendant huit semaines dans des bacs circulaires en béton. Quatre densités de mise en charge ont été testées en triplicat. 
Les densités ont été de 10, 15, 20 et 25 alevins $/ \mathrm{dm}^{3}$ d'eau. L'aliment utilisé a été le Coppens ( $45 \%$ de protéines, $10 \%$ de lipides et $18,5 \mathrm{Kj} / \mathrm{g}$ d'Énergie Brute). La ration alimentaire a été de $4 \%$ de la biomasse/bassin/J. L'aliment a été distribué manuellement chaque jour toutes les deux heures entre 8 et 18 heures. La température moyenne de l'eau a été de $27,21 \pm 0,13^{\circ} \mathrm{C}$. Le pH et le taux moyen d'oxygène dissous de l'eau ont été respectivement de $5,45 \pm 0,26$ et $5,48 \pm 0,32 \mathrm{mg} / \mathrm{L}$. Des pêches de contrôle ont été faites chaque semaine afin d'estimer l'évolution du poids moyen et d'ajuster la ration à la biomasse. La biomasse totale de tous les alevins par bassin a été alors mesurée à chaque pêche de contrôle. $\mathrm{Au}$ début et à la fin de l'expérience, le nombre total d'alevins et la biomasse par bassin ont été mesurés. Les poids et longueurs individuels de tous les poissons ont été également mesurés par bassin.

Paramètres calculés et analyses statistiques :

Les paramètres suivants ont été calculés pour chaque traitement :

Taux de croissance spécifique (TCS \%/J) $=100(\mathrm{LnPf}-$ $\mathrm{LnPi} / \Delta \mathrm{t}$ où $\mathrm{LnPf}=$ logarithme népérien du poids final, $\mathrm{LnPi}=$ logarithme népérien du poids initial, $\Delta \mathrm{t}=$ durée de l'expérience;

\section{RÉSULTATS}

Les résultats de l'effet de la densité de mise en charge des alevins de $P$. obscura sur leurs performances zootechniques et la production sont présentés dans le tableau 1. Aucune mortalité n'a été enregistrée au cours de cette étude. La densité de mise en charge n'a pas alors eu un effet significatif sur le taux de survie des alevins de $P$. obscura $(P>0,05)$. Par contre, le taux de croissance spécifique (figure 1) a significativement varié avec la densité de mise en charge des alevins $(P<0,05)$. La croissance s'est améliorée au fur et à mesure que la densité de mise en charge a augmenté. Le poids moyen final et le taux de croissance spécifique les plus élevés $(P$ $<0,05)$ ont été alors obtenus avec les densités 20 et 25 alevins $/ \mathrm{dm}^{3}$. Le facteur de condition a significativement varié $(P<0,05)$ de $0,76 \pm 0,04\left(10\right.$ alevins $\left./ \mathrm{dm}^{3}\right)$ à $0,88 \pm$
Efficacité Alimentaire $(\mathrm{EA})=(\mathrm{Bf}-\mathrm{Bi}) / \mathrm{RD}$ où $\mathrm{Bf}=$ Biomasse finale, $\mathrm{Bi}=$ Biomasse initiale, $\mathrm{RD}=$ Ration Distribuée ;

Taux de Survie $($ TS en $\%)=100 \times$ Nf $/ \mathrm{Ni}$ où Nf $=$ Nombre final, $\mathrm{Ni}=$ Nombre initial;

Coefficient de condition $(\mathrm{K}$ en $\%)=\left(\mathrm{Pf} / \mathrm{L}^{3}\right) \times 100$ où $\mathrm{Pf}=$ Poids final, $\mathrm{L}=$ longueur totale; Production $(\mathrm{P})=(\mathrm{Bf}-$ $\mathrm{Bi}) / \mathrm{V}$, Production annuelle $(\mathrm{Pa})=(\mathrm{Bf}-\mathrm{Bi}) \times 52$ semaines $/ \mathrm{Vx}$ Durée de l'expérience où $\mathrm{Bf}=$ Biomasse finale, $\mathrm{Bi}=$ Biomasse initiale, $\mathrm{V}=$ Volume d'eau.

L'analyse statistique a été effectuée à l'aide du logiciel Statistica (version 5.5) par la méthode d'analyse de la variance à un critère de classification (ANOVA 1). Le facteur fixe considéré dans le modèle ANOVA 1 a été la densité de mise en charge. Le test de Hartley nous a permis de tester l'homogénéité des variances (Dagnelie, 1975). Le test LSD (Least Significant Difference) (Saville, 1990) a été utilisé pour apprécier les différences entre les traitements pour chaque paramètre zootechnique calculé. Un seuil de probabilité de $5 \%$ a été retenu. Les moyennes obtenues pour chaque paramètre ont été présentées \pm l'écart type.

$0,04\left(25\right.$ alevins $\left./ \mathrm{dm}^{3}\right)$. Les facteurs de condition les plus élevés ont été alors obtenus avec les plus fortes densités de mise en charge étudiées. L'efficacité alimentaire a significativement augmenté lorsque la densité de mise en charge croit $(P<0,05)$. L'efficacité alimentaire la plus faible a été obtenue chez les alevins stockés à la densité de $10 / \mathrm{dm}^{3}(P<0,05)$. La production c'est-à-dire le gain de biomasse par volume d'eau a été significativement influencé par la densité de mise en charge $(P<0,05)$. Les productions obtenues avec la densité de 25 alevins $/ \mathrm{dm}^{3}$ ont été plus élevées que toutes les autres. Le gain de biomasse (production) chez les alevins stockés à la densité de 25 alevins $/ \mathrm{dm}^{3}$ a été plus de trois fois plus élevé que celui observé avec la densité de $10 / \mathrm{dm}^{3}(P<$ 0,05). 


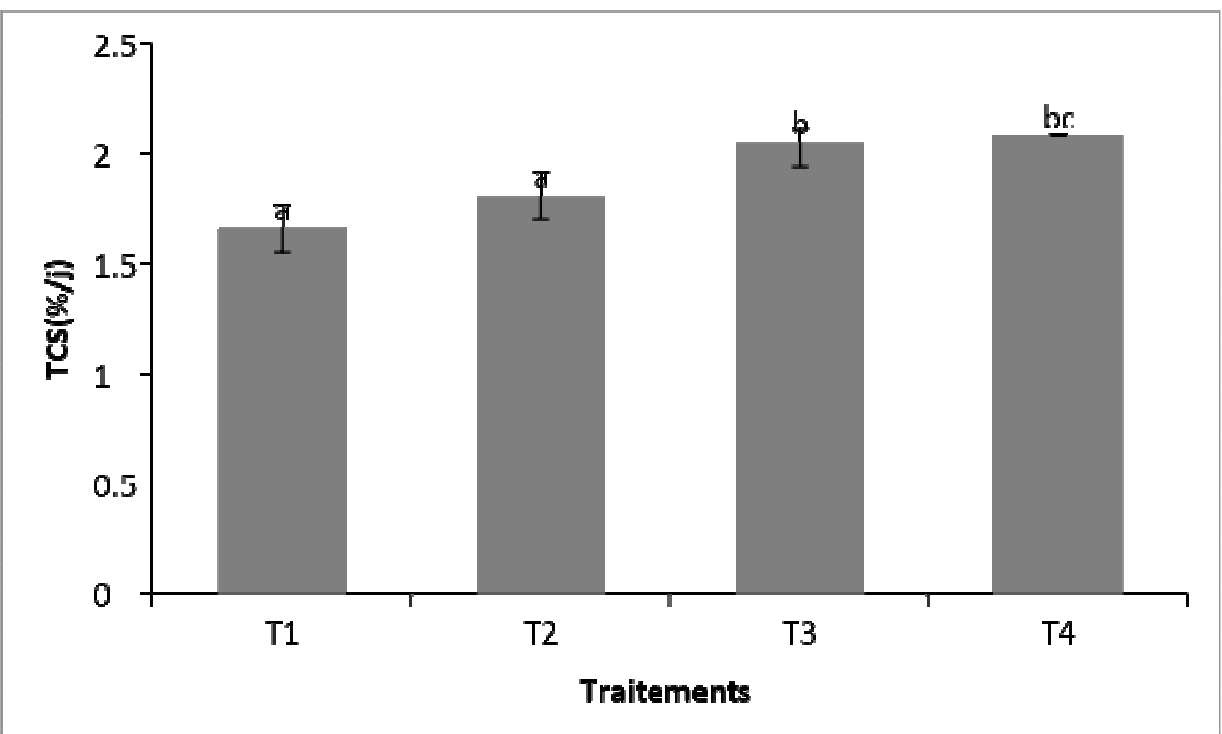

Figure 1 : Variation du taux de croissance spécifique en fonction des traitements

Tableau 1 : Paramètres zootechniques des alevins de $P$. obscura stockés à des densités différentes

\begin{tabular}{|c|c|c|c|c|}
\hline $\begin{array}{l}\text { Densités (nombre } \\
\text { d'alevins/dm³) }\end{array}$ & 10 & 15 & 20 & 25 \\
\hline Poids moyen initial (g) & $9,98 \pm 1,00^{a}$ & $9,59 \pm 0,41^{a}$ & $9,31 \pm 0,69 \mathrm{a}$ & $9,45 \pm 0,08^{a}$ \\
\hline Poids moyen final (g) & $22,09 \pm 1,19 a$ & $22,87 \pm 0,69$ a & $24,85 \pm 0,52^{b}$ & $25,62 \pm 0,66$ bc \\
\hline Taux de survie (\%) & $100^{a}$ & $100^{a}$ & $100^{a}$ & 100 a \\
\hline Efficacité alimentaire & $0,75 \pm 0,02^{a}$ & $0,85 \pm 0,05^{b}$ & $0,96 \pm 0,02^{c}$ & $1,13 \pm 0,03^{d}$ \\
\hline Facteur de condition (\%) & $0,76 \pm 0,04 a b$ & $0,78 \pm 0,04 \mathrm{~b}$ & $0,87 \pm 0,04 c$ & $0,88 \pm 0,04 c$ \\
\hline Production $\left(\mathrm{g} / \mathrm{dm}^{3}\right)$ & $121,10 \pm 4,06^{a}$ & $199,27 \pm 12,12^{b}$ & $310,77 \pm 3,70^{c}$ & $404,34 \pm 17,33^{d}$ \\
\hline Production ( $\left.\mathrm{g} / \mathrm{dm}^{3} / \mathrm{an}\right)$ & $794,72 \pm 26,64^{a}$ & $1307,71 \pm 79,54^{b}$ & $2039,43 \pm 24,28^{c}$ & $2653,48 \pm 113,73^{d}$ \\
\hline
\end{tabular}

Les valeurs se trouvant dans la même colonne et partageant la même lettre ne sont pas significativement différentes $(P>$ $0,05)$.

\section{DISCUSSION}

La densité de mise en charge des poissons est un facteur déterminant en aquaculture. C'est un indicateur important qui détermine la viabilité technique et économique d'une entreprise aquacole (Pangni et al., 2008 ; Gao et al., 2017). En effet, elle affecte significativement la croissance et la maturation des poissons quel que soit le système d'élevage utilisé (Rahman et al., 2005 ; Celada et al., 2007 ; Narejo et al., 2010 ; Hannibal et al., 2011). Afin d'avoir des productions optimales et de tirer un maximum de profit, il faudra alors élever les poisons à des densités de mise en charge optimales. Les taux de survie $(100 \%)$ obtenus montrent que les alevins de $P$. obscura sont résistants au stress dû aux manipulations en milieu contrôlé. Cette caractéristique constitue un atout non négligeable et l'un des critères que doit remplir un bon poisson d'élevage. Les performances zootechniques à savoir le poids moyen final, le taux de croissance spécifique, le coefficient de condition et l'efficacité alimentaire s'améliorent lorsque la densité de mise en charge augmente et leurs valeurs les plus élevées s'obtiennent avec les plus fortes densités utilisées au cours de notre étude. Nos observations corroborent avec celles de Qin et Fast (1998) et Muthmainnah (2013) qui ont montré une amélioration des performances zootechniques des alevins de Channa striata (Channidae asiatique) au fur et à mesure que la densité de mise en charge augmente. Chez plusieurs espèces, les paramètres zootechniques varient en fonction de la densité de mise en charge des poissons (Khattab et al., 2004 ; Abou et al., 2007; Imorou Toko et al., 2008; Gaar et al., 2011; M'Balaka et al., 2012; Chattopadhay et al., 2012 ; Hasanalipour et al., 2013). Le stockage des poissons à des densités inadéquates constitue un facteur de stress chronique qui réduit leurs 
performances de croissance à cause des effets de leurs interactions sociales pour avoir accès à l'aliment et à l'espace (Sugunan et Katiha, 2004). Ces comportements favorisent une diminution de l'appétit et la perte d'une part importante de l'énergie normalement destinée à la croissance et par conséquent, une réduction du taux de croissance spécifique et de l'efficacité alimentaire (Pankhurst et Van der kraak, 1997 ; El Sayed, 2002). L'augmentation de la densité de mise en charge jusqu'à un niveau donné réduit l'agressivité et permet l'amélioration des performances zootechniques de certaines espèces piscicoles (Conte et al., 2008; Chattopadhyay et al., 2012). Nos résultats sont conformes à ceux de Sampath et Pandian (1984) d'une part et de Qin et Fast (1998) d'autre part, qui ont montré une amélioration des performances zootechniques des alevins de $C$. striata au fur et à mesure que la densité de leur mise en charge augmente. Imorou Toko et al. (2008), Saoud et al. (2007), Jamabo et Keremah (2009), Edward et al. (2010) et Jiwyan (2011) ont aussi fait des observations pareilles chez plusieurs espèces de poissons chats. Cette similitude entre l'aptitude des Channidae et celle des poissons chats à supporter des fortes densités d'élevage se justifie par le fait qu'ils possèdent tous un organe accessoire de respiration supra branchial qui leur permet de vivre dans des milieux peu oxygénés (Liem, 1980 ; Hecht et Uys, 1997). Le coefficient de condition représente un indicateur de l'embonpoint des espèces en aquaculture. Les meilleurs

\section{CONCLUSION}

Les performances de croissance (poids moyen final, taux de croissance spécifique, coefficient de condition), d'utilisation de l'aliment (efficacité alimentaire) et la production des alevins de $P$. obscura augmentent au fur et à mesure que la densité de mise en charge croît. Les

\section{REMERCIEMENTS}

Nous remercions le Ministère de l'Enseignement Supérieur et de la Recherche Scientifique de la

\section{RÉFÉRENCES BIBLIOGRAPHIQUES}

Abou Y, Fiogbe ED, Micha JC. 2007. Effects of stocking density on growth, yield and profitability of farming Nile tilapia Oreochromis niliticus, L. fed Azolla diet, in earthen ponds. Aquaculture Research. 38, 585 - 604 .

Aksungur N, Aksungur M, Akbulut B, Kutlu I. 2007. Effects of stocking density on growth performance, survival and food conversion ration of turbot (Psetta maxima) in the net cages coefficients de condition s'observent lorsque l'environnement est favorable au développement des poissons (Blackwell et al., 2000). Nos résultats montrent que les valeurs les plus élevées significativement pour ce paramètre sont obtenues avec les densités 20 et 25 alevins/ $\mathrm{dm}^{3}$. II est d'environ $0,88 \%$ et est proche des résultats obtenus par Vodounnou et al. (2016) chez les alevins de $P$. obscura élevés en milieu contrôlé. Le taux de croissance spécifique le plus élevé $(2,08 \% / \mathrm{J})$ observé avec la densité de 25 alevins $/ \mathrm{dm}^{3}$ est proche des 2,55\%/J issus des travaux de Kpoguè et al. (2013b) chez les alevins de $P$. obscura. Ce résultat est semblable à ceux de Aliyu -Paiko et al. (2010) et Zehra et Khan (2011) qui ont obtenu des taux de croissance spécifique de 2,56 et $1,82 \% / J$ respectivement chez les alevins de Channa striatus et de C.punctatus nourris avec des aliments secs et élevés en milieu contrôlé. Nos résultats montrent que la production augmente avec la densité de mise en charge des alevins de $P$. obscura. Morengoni (2006) et Phan et al. (2009) ont aussi remarqué une amélioration de la production lorsque la densité augmente quel que soit le système d'élevage utilisé. Ce résultat est l'implication logique de l'amélioration des performances de croissance observée lorsque la densité de stockage des alevins augmente. En effet, la production étant le résultat du gain de biomasse par volume d'eau, elle est positivement corrélée aux paramètres de croissance des poissons. Ainsi, toute amélioration de la croissance entraîne celle de la production.

meilleurs résultats ont été obtenus avec la densité de stockage de 25 alevins $/ \mathrm{dm}^{3}$. Nous suggérons alors une densité de mise en charge de 25 alevins de $P$. obscura/ $\mathrm{dm}^{3}$ élevés en milieu contrôlé afin de garantir une bonne rentabilité aux pisciculteurs.

République du Bénin qui a financé cette étude à travers son Programme d'Incitation à la Fonction Enseignante.

on the Southeastern coast of the Black sea. Turkish Journal of Fisheries and Aquatic Sciences. 7: $147-152$.

Aliyu - Paiko M, Hashim R, Shu - Chien AC. 2010. Influence of dietary lipid/protein ratio on survival, growth, body indices and digestive lipase activity in Snakehead (Channa striatus, Bloch 1793) fry reared in re - circulating water system. Aquaculture Nutrition. $16: 466-474$. 
Blackwell BG, Brown ML, Willis DW. 2000. Relative weight $(\mathrm{Wr})$ status and current use in fisheries assessment and management. Revue of Fisheries Sciences. 8: 1-44.

Bolaji BB, Mfon TU, Utibe DI. 2011. Preliminary study on the aspects of the biology of snakehead fish Parachanna obscura (Günther) in a Nigerian wetland. African Journal of Food, Agriculture, Nutrition and Development. 11 (2) : 4708-4717

Bonou CA and Teugels GG. 1985. Révision systématique du genre Parachanna Teugels et Daget, 1984 (Pisces, Channidae). Revue d' Hydrobiologie Tropicale. 18(4) : 267-280.

Cahu C, Salen P, de Lorgeril M. 2004. Farmed and wild fish in the prevention of cardiovascular diseases : assessing possible differences in lipid nutritional values. Nutrition Metabolism and Cardiovascular Diseases. 14: 34-41.

Celada JD, Aguilera A, Carral JM, Saez - Royuela M, Melendre PM, Perez JR. 2007. Effects of stocking density on survival and growth of juvenile tench (Tinca Tinca L). Aquaculture International. 15: 461 - 465 .

Chattopadhyay DN, Mohapatra BC, Adhikari S, Pani KC, Jena JK, Eknath AE. 2012. Effects of stocking density of Labeo rohita on survival, growth and production in cages. Aquaculture International. DOI 10.1007/s10499-012-9528-2.

Conte L, Sonoda DY, Shirota R, Cyrino JEP. 2008. Productivity and economics of Nile Tilapia Oreochromis niloticus cage culture in SouthEast Brazil. Journal of Applied Aquaculture. 20:18-37.

Dagnelie P. 1975. Théorie et Méthodes Statistiques (Vol. II). Presses Agronomiques de Gembloux: Belgique ; $463 \mathrm{p}$.

Edward A, Ladu BMB, Elihu A. 2010. Growth, survival and production economics of Clarias gariepinus fingerlings at different stocking densities in concrete tanks. African Journal of General Agriculture. 6 (2) : $59-66$.

El-sayed AFM. 2002. Effect of stocking density and feeding levels on growth and feed efficiency of Nile tilapia Oreochromis niloticus. Aquacult. Res., 33: $621-626$.

El-Sherif MS and El-Feky AMI. 2009. Performance of Nile tilapia (Oreochromis niloticus) fingerlings. I. Effect of $\mathrm{pH}$. International Journal of Agriculture and Biology. 11: 297-300.

Fiogbé ED and Kestemont P. 2003. Optimum daily ration for Eurasian perch Perca fluviatilis L. reared at its optimum growing temperature. Aquaculture. 216: $243-252$.

Gao Y, He Z, Vector H, Zhao B, Li Z, He J, Lee JY, Chu Z. 2017 .Effect of Stocking Density on Growth, Oxidative Stress and HSP 70 of Pacific White Shrimp Litopenaeus vannamei. Turkish Journal of Fisheries and Aquatic Sciences 17: 877-884. DOI : 10.4194/1303-2712-v17_5_04

Garr AL, Lopez H, Pierce R, Davis M. 2011. The effect of stocking density and diet on the growth and survival of cultured Florida apple snails, Pomacea paludosa. Aquaculture. 311, 139 145.

Günther A. 1861. Catalogue of the Acanthopterygian fishes. The Trustees, London. Vol. III, $586 \mathrm{p}$.

Hannibal MC, Amparo LF, Aurelio AC. 2011. Effect of Stocking Density on Growth Performance, Survival and Production of Silver Pompano, Trachinotus blochii, (Lacépède, 1801) in Marine Floating Cages, Asian Fisheries Science. 24:321-330.

Hasanalipour A, Eagderi S, Poorbagher H, Bahman M. 2013. Effects of stocking density on blood cortisol, glucose and cholesterol levels of immature Siberian sturgeon (Acipenser baerii Brandt, 1806). Turkish Journal of Fisheries and Aquatic Sciences. 13: 27 - 32. DOI 10.4194/1303-2712-V13-1-04.

Hecht T and Uys W. 1997. Effect of density on the feeding and aggressive behaviour in juvenile African catfish, Clarias gariepinus. South African Journal of Sciences. 93: 537 - 541.

Imorou Toko I, Fiogbé ED, Kestemont P. 2008. Determination of appropriate age and stocking density of vundu larvae, Heterobranchus longifilis (Valenciennes 1840), at the weaning time. Aquaculture Research. 39, 24 - 32.

Jamabo NA and Keremah RI. 2009. Effects of stocking density on the growth and survival of the fingerlings of Clarias gariepinus (Burchell, 1822). Journal of Fisheries International. 4 (4) : $55-57$.

Jiwyam W. 2011. The effect of stocking density on yield, growth, and survival of Asian river catfish (Pangasius bocourti Sauvage, 1880) cultured in cages. Aquaculture International. 19: 987-997

Khattab YAE, Abdel-Tawwab M, Ahmad MH. 2004. Effect of protein level and stocking density on growth performance, survival rate, feed utilization and body composition of Nile tilapia fry (Oreochromis niloticus L.). In: New Dimension in Farmed 
Tilapia, Proceedings of the Sixth International Symposium on Tilapia in Aquaculture. (Eds. R.B. Bolivar, G.C. Mair and K. Fitzsimmons), Manila, Philippines, (1) :264-276.

Kpoguè D and Fiogbé E. 2012. Feeding rate requirements for Parachanna obscura fry reared under controlled environmental conditions. Journal of Applied Biosciences. 55: 3962-3972.

Kpoguè Diane NS, Houankanlin Narcisse, Dougnon Jacques, Fiogbé Emile. 2013c. Effet de la teneur en lipides des aliments sur les performances zootechniques des alevins de Parachanna obscura. Résumé présenté au 4ème Colloque des Sciences, Cultures et Technologies de I'UAC - Bénin, 23 - 28 Septembre 2013, pp 662.

Kpoguè DNS, Ayanou GA, Imorou Toko I, Mensah GA, Fiogbé ED. 2013b. Influence of dietary protein levels on growth, feed utilization and carcass composition of snakehead Parachanna obscura (Günther, 1861) fingerlings. International Journal of Fisheries and Aquaculture. 5(5):71 - 77.

Kpoguè DNS, Mensah GA, Fiogbé ED. 2013a. A review of biology, ecology and prospect for aquaculture of Parachanna obscura. Review in Fish Biology and Fisheries. 23 (1) : 41 -50. DOI. 10.1007/s 11160-012-9281-7.

Leatherland JF and Cho CY. 1985. Effect of rearing density on thyroid and interregnal gland activity and plasma and hepatic metabolite levels in rainbow trout, Salmo gairdneri. Richardson. Journal of Fisheries and Biology. 27: 583 - 592.

Liem K. 1980. Air breathing in advanced teleosts : Biochemical and evolutionary aspects. In : Environment Physiology of Fishes. Ed. M.A. Ali. Plenum Press, New York. 57-91.

M'Balaka M, Kassam D, Rusuwa B. 2012. The effect of stocking density on the growth and survival of improved and unimproved strains of Oreochromis shiranus. Egyptian Journal of Aquatic Research. 38.205-211 DOI 10.1016/j.ejar.2012.12.013.

Ma A, Chen C, Lei J, Chen S, Zhuang Z, Wang Y. 2006. Turbot Scophthalmus maximus: stocking density on growth, pigmentation and feed conversion. Chineese Journal of Oceanography and Limnology. 24 (3) : $307-312$.

Melard C, Baras E, Mary L, Kestemont P. 1995. Reltionships between growth, cannibalism and survival rate in intensively cultured larvae and alevins of perch (Perca fluviatilis). Abstract
Percis II, Second International Percid fish Symposium. Vaasa, Finland, 21-25 August 1995, pp : 53-54.

Morengoni NG. 2006. Production of the Nile tilapia Oreochromis niloticus (Chitralada strain) reared in cages with different stocking densities. Archeology and Zootechny. 55:127-138.

Muthmainnah D. 2013. Growout of striped snakehead (Channa striata) in swamp water system using fences and cages. $4^{\text {th }}$ International Conference on Biology, Environment and Chemistry. IPCBEE. Vol 58: 52-55. IACSIT Press, Singapore DOI : 10.7763/IPCBEE 2013

Pangni K, Atsé BC, Kouassi NJ. 2008. Effect of stocking desity on growth and survival of the African catfish Chrysichthys nigrodigitatus, Claroteidae (Lacépède 1803) larvae in circular tanks. Living Research and Rural Development. 20 (7). 8p.

Pankhurst NW. And Van der Kraak G. 1997. Effects of stress on reproduction and growth of fish. In : Fish Stress and Health in Aquaculture (ed. By J.K. Iwama \& A.D. Pickering) Cambridge University Press, Cambridge, UK. 73-93.

Phan LT, Bui TM, Nguyen TTT, Gooley GJ, Ingram BA, Nguyen HV, Nguyen PT, De Silva SS. 2009. Current status of farming practices of striped catfish, Pangasianodon hypophthalmus in the Mekong Delta, Vietnam. Aquaculture. Doi: 10.1016/j.aquaculture.2009.08.017.

Qin JG and Fast AW. 1998. Effects of temperature, size and density on culture performance of snakehead, Channa striatus (Bloch), fed formulated feed. Aquaculture Research. 29: 299 -303 .

Saoud IP, Ghanawi J, Lebbos N. 2008 Effects of stocking on the survival, growth, size variation and condition index of juvenile rabbitfish Siganus rivulatus. Aquaculture International 16(2) : 109116, DOI 10.1007/s10499-007-9129-7.

Saville DJ. 1990. Multiple comparison procedures : the practical solution. American Statistic, 44 (2) : $174-180$.

Schram E., Van der Heul JW., Kamstra A., Verdergem MCJ. 2006. Stocking density dependent growth of dover (Solea solea). Aquaculture. 252: 239 247.

Sugunan W and Katiha P. 2004. Impact of stocking density on yield in small reservoirs in andra Pradesh. Indian Fisheries Management and Ecology. 11:193 - 202. 
Tan C, Sun D, Tan H, Liu W, Luo G, Wei X. 2018. Effects of Stocking Density on Growth, Body Composition, Digestive Enzyme Levels and Blood Biochemical Parameters of Anguilla marmorata in a Recirculating Aquaculture System. Turkish Journal of Fisheries and Aquatic Sciences.18 : 9-16. DOI : 10.4194/13032712-v18_1_02

Vodounnou DSJ, Kpogue DNS, Mensah GA, Fiogbe ED. 2016. Culture of earthworms (Eisenia fetida), production, nutritive value and utilization of its meal in diet for Parachanna obscura fingerlings reared in captivity. International Journal of Fisheries and Aquatic Sciences 4(5) : 01-05.

Wirat J. 2011. The effect of stocking density on yield, growth, and survival of Asian river catfish (Pangasius bocourti Sauvage, 1880) cultured in cages. Aquaculture International. 19:987-997.

Zehra S and Khan MA. 2011. Dietary protein requirement for fingerling Channa punctatus (Bloch), based on growth, feed conversion, protein retention and biochemical composition. Aquaculture International. DOI 10.1007/s10499-011-9470-8. 